九州大学学術情報リポジトリ

Kyushu University Institutional Repository

\title{
Enhanced liver functions in mouse hepatoma cells by induced overexpression of liver- enriched transcription factors
}

Yamamoto, Hideaki

Department of Chemical Engineering, Faculty of Engineering, Kyushu University

Kawabe, Yoshinori

Department of Chemical Engineering, Faculty of Engineering, Kyushu University

Ito, Akira

Department of Chemical Engineering, Faculty of Engineering, Kyushu University

Kamihira, Masamichi

Department of Chemical Engineering, Faculty of Engineering, Kyushu University

http://hdl. hand le. net/2324/27294

出版情報: Biochemical Engineering Journal. 60，pp.67-73，2012-01-15. Elsevier バージョン:

権利関係: (C) 2011 Elsevier B.V. 


\title{
Enhanced liver functions in mouse hepatoma cells by induced overexpression of liver-enriched transcription factors
}

\author{
Hideaki Yamamoto, Yoshinori Kawabe, Akira Ito, and Masamichi Kamihira* \\ Department of Chemical Engineering, Faculty of Engineering, Kyushu University, 744 Motooka, \\ Nishi-ku, Fukuoka 819-0395, Japan
}

*Corresponding author. E-mail: kamihira@chem-eng.kyushu-u.ac.jp

Phone: +81-92-802-2743; Fax: +81-92-802-2793 


\begin{abstract}
Hepatoma cells, which are derived from liver carcinoma, are able to proliferate infinitely under culture conditions. However, the liver functions of hepatoma cells are generally low compared with those of hepatocytes in a liver. Here, we attempted to create genetically engineered hepatoma cells with enhanced liver functions by overexpression of liver-enriched transcription factors (LETFs), which are associated with the transcription of liver-specific genes and hepatic differentiation. For this purpose, genes for eight LETFs, hepatocyte nuclear factor (HNF)-1 $\alpha, \mathrm{HNF}-1 \beta, \mathrm{HNF}-3 \beta, \mathrm{HNF}-4 \alpha$, HNF-6, CCAAT/enhancer binding protein (C/EBP)- $\alpha, \mathrm{C} / \mathrm{EBP}-\beta$ and $\mathrm{C} / \mathrm{EBP}-\gamma$, were obtained from the mouse liver. Mouse hepatoma Hepa1-6 cells were transduced with retroviral vectors, in which inducible expression cassettes for the LETF genes were introduced. Cell clones with inducible expression of high liver functions were established. Upon overexpression of the LETF genes, cell proliferation ceased and the cells exhibited an epithelial morphology, indicating hepatic maturation of hepatoma cells. This approach for genetic modification of hepatoma cells may be promising for the construction of cells for use in bioartificial liver support systems.
\end{abstract}

Keywords: Animal cell culture; Biomedical; Recombinant DNA; Hepatoma cell; Liver-enriched transcription factor; Liver function 


\section{Introduction}

Liver failure is one of the major causes of death around the world [1], because the liver is multifunctional and a vital organ for survival. Although liver transplantation is the only effective treatment for acute liver failure [2], it has several limitations including the shortage of organ donors. Recently, bioartificial liver (BAL) support systems, in which functional hepatic cells such as primary hepatocytes are placed in a bioreactor to cover the hepatic functions through blood circulation between the bioreactor and a liver failure patient [3], have been expected to make up for the organ donor shortage.

Although normal human hepatocytes are the ideal cell source for BAL systems, it is difficult to culture primary hepatocytes for long periods of time with the current cell culture technology and to obtain sufficiently large numbers of differentiated human hepatocytes for practical application of BAL systems. Therefore, embryonic stem cells, hepatic stem cells and bone marrow cells, which possess proliferation ability and retain their differentiation potential, have been considered as alternative cell sources for large-scale hepatocyte preparation [4]. However, the efficiency and degree of differentiation in these cells under culture conditions are not presently high and are insufficient to obtain large numbers of functional hepatocytes. Moreover, cultures for the proliferation and differentiation of such stem cells using cytokines are often time-consuming and costly processes.

Hepatoma cells derived from liver carcinoma are one of the alternative cell sources for BAL systems. Hepatoma cells can be cultured easily and inexpensively compared with the stem cells described above. Hepatoma cells also express some liver functions under ordinary culture conditions, because they are derived from hepatocytes. However, the expression levels of the liver functions in hepatoma cells are generally very low compared with those of hepatocytes in a liver.

Liver-enriched transcription factors (LETFs) play critical roles in liver development and maintenance of the hepatocyte phenotype through transcriptional regulation of liver-specific gene 
expressions. Several LETFs are always expressed in hepatocytes and their combined actions appropriately control the gene expressions that are necessary to maintain various hepatic functions, such as albumin secretion, urea synthesis and drug metabolisms, at the transcriptional level [5]. In the present study, we attempted to create genetically engineered hepatoma cells with enhanced liver functions by overexpression of LETFs. For this purpose, we selected eight transcription factors, hepatocyte nuclear factor (HNF)-1 $\alpha$, HNF-1 $\beta$, HNF-3 $\beta$, HNF-4 $\alpha$, HNF-6, CCAAT/enhancer binding protein (C/EBP)- $\alpha, \mathrm{C} / \mathrm{EBP}-\beta$ and $\mathrm{C} / \mathrm{EBP}-\gamma$, based on their known functions in hepatocytes [6,7], and inducible expression cassettes for these LETF genes were introduced into hepatoma cells by retroviral transduction.

\section{Materials and Methods}

\subsection{Cell culture}

Mouse hepatocellular carcinoma Hepa1-6 cells (Riken BioResource Center, Tsukuba, Japan) were grown in high-glucose Dulbecco's modified Eagle's medium (DMEM) (Sigma-Aldrich, St. Louis, MO, USA) supplemented with $10 \%$ fetal bovine serum (FBS), $0.1 \mathrm{mg} / \mathrm{mL}$ streptomycin sulfate and $100 \mathrm{U} / \mathrm{mL}$ penicillin G potassium (Wako Pure Chemical Industries, Osaka, Japan). 293FT cells (Invitrogen, Carlsbad, CA, USA) were used as producer cells for retroviral vectors based on mouse stem cell virus (MSCV) [8], and were cultured in high-glucose DMEM supplemented with 10\% FBS, 0.1 mM MEM non-essential amino acids (Invitrogen) and 20 mM HEPES (Dojindo, Kumamoto, Japan) in collagen-coated tissue culture dishes (Asahi Techno Glass, Tokyo, Japan). Parenchymal hepatocytes were obtained from female C57BL/6NCrSlc mice ( 8 weeks old) by the collagenase perfusion method. The animal experiment was approved by the Ethics Committee for Animal Experiments of the Faculty of Engineering, Kyushu University (A23-146-0). Hepatocytes were seeded into the wells of 24 -well collagen-coated plates (Asahi Techno Glass) at a density of $1.0 \times 10^{5}$ cells/well and cultured in Williums' medium E (Invitrogen) supplemented with 5\% FBS, $0.1 \mu \mathrm{M}$ 
$\mathrm{CuSO}_{4} 5 \mathrm{H}_{2} \mathrm{O}, 25 \mathrm{nM} \mathrm{Na}_{2} \mathrm{SeO}_{3}, 1.0 \mu \mathrm{M}$ dexamethasone, $0.1 \mu \mathrm{M}$ insulin, $20 \mathrm{ng} / \mathrm{mL}$ EGF (Biomedical Technologies, Stoughton, MA, USA), $0.1 \mathrm{mg} / \mathrm{mL}$ streptomycin sulfate and $100 \mathrm{U} / \mathrm{mL}$ penicillin G potassium. Liver functions of hepatocytes were measured on day 1-2 of culture. All cells were cultured at $37^{\circ} \mathrm{C}$ in a $5 \%(\mathrm{v} / \mathrm{v}) \mathrm{CO}_{2}$ incubator.

\subsection{Cloning of mouse LETFs and construction of retroviral vector plasmids}

Total RNA was extracted from the mouse liver using a commercially available kit (RNAiso plus; Takara, Otsu, Japan) according to the manufacturer's protocol. The isolated RNA was reverse-transcribed with an oligo-dT primer using ReverTra Ace reverse transcriptase (Toyobo, Osaka, Japan) to produce cDNA. The coding regions of the mouse LETF genes were amplified by PCR from the cDNA using KOD-plus DNA polymerase (Toyobo) and the primer pairs listed in Supplementary Table 1. The primers included restriction site sequences (forward primers, XhoI or SalI site; reverse primers, BamHI site) to append them to the ends of each PCR product to facilitate subsequent subcloning. The PCR amplifications were performed at $94^{\circ} \mathrm{C}$ for $2 \mathrm{~min}$, followed by 35 cycles of amplification at $94^{\circ} \mathrm{C}$ for $15 \mathrm{~s}$, various annealing temperatures (Supplementary Table 1) for $30 \mathrm{~s}$ and $68^{\circ} \mathrm{C}$ for $60-90 \mathrm{~s}$. For HNF-1 $\alpha$, the amplification of the sequence was divided into two parts because of the length of the sequence. The PCR products of LETFs except for HNF-1 $\alpha$ and HNF-4 $\alpha$ were digested with XhoI and BamHI and ligated into XhoI- and BamHI-digested pBluescriptII KS (-) (Toyobo). The PCR products of HNF-1 $\alpha$ and HNF-4 $\alpha$ were digested with SalI and BamHI and ligated into SalI- and BamHI-digested pBluescriptII KS (-). The DNA sequences of the PCR products were confirmed using a DNA sequencer (Prism 3130 Genetic Analyzer; Applied Biosystems, Foster City, CA, USA) and compared with their sequences in the GenBank database.

MSCV-based retroviral vectors were used for gene transfer into Hepa1-6 cells. The Tet-On system (Clontech, Mountain View, CA, USA) was incorporated into the retroviral vectors for inducible expression of the LETF genes. For retroviral vector production, retroviral vector plasmids encoding 
expression cassettes for the reverse tetracycline-dependent transactivator (rtTA) and LETF genes were constructed. The pQMSCV/EGFP-CMV-rtTA-WPRE plasmid encoded a constitutive expression cassette for rtTA that was activated by the addition of doxycycline (Dox). The pQMSCV/EGFP-TREtight-LETF-WPRE plasmids encoded an expression cassette for each LETF including a tetracycline-responsive element, such that LETF expression was promoted by the activated rtTA. These plasmids included an enhanced green fluorescent protein (EGFP) gene under the control of viral LTR promoters for evaluation of the viral titer. To construct pQMSCV/EGFP-CMV-rtTA-WPRE, a DNA fragment of the EGFP gene was amplified from pIRES-EGFP (Clontech) by PCR using the primers

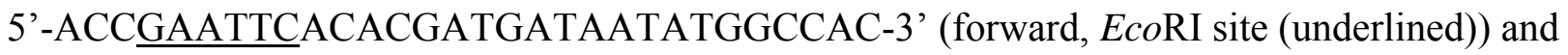
5'-ATTCTCGAGAGGCCGCTTTACTTGTACAG-3' (reverse, XhoI site (underlined)). The PCR fragment was ligated into EcoRI- and XhoI-digested pQMSCV/CMV-VEGF-IRES-EGFP [9] to generate $\mathrm{pQMSCV/EGFP.} \mathrm{Next,} \mathrm{the} \mathrm{woodchuck} \mathrm{hepatitis} \mathrm{virus} \mathrm{posttranscriptional} \mathrm{regulatory}$ element (WPRE) sequence from pMSCV/G $\triangle \mathrm{AhEpoW} \mathrm{[10]} \mathrm{was} \mathrm{inserted} \mathrm{into} \mathrm{ClaI-digested}$ pQMSCV/EGFP, resulting in pQMSCV/EGFP-WPRE. Thereafter, pQMSCV/EGFP-CMV-rtTA-WPRE was generated by ligation of the cytomegalovirus (CMV) promoter and the rtTA sequence from pTet-On Advanced (Clontech) into XhoI- and ClaI-digested pQMSCV/EGFP-WPRE. For the construction of pQMSCV/EGFP-TREtight-LETF-WPRE, the tetracycline-responsive promoter from pTRE-Tight (Clontech) was ligated into XhoI- and ClaI-digested pQMSCV/EGFP-WPRE to generate pQMSCV/EGFP-TREtight-WPRE. The pQMSCV/EGFP-TREtight-LETF-WPRE plasmids were then generated by ligation of the XhoI- and BamHI-digested DNA fragments encoding each LETF gene into XhoI- and BamHI-digested pQMSCV/EGFP-TREtight-WPRE. pQMSCV/EGFP-TREtight-DsRed-WPRE was constructed by insertion of a DNA fragment encoding DsRed protein from pIRES2-DsRed-Express2 (Clontech) into XhoI- and BamHI-digested pQMSCV/EGFP-TREtight-WPRE. 


\subsection{Retroviral vector production and infection}

Retroviral vectors encoding the rtTA and LETF genes were produced as described previously [11]. Culture medium containing retroviral particles was filtered to remove cell debris using a $0.45-\mu \mathrm{m}$ cellulose acetate filter (Advantec, Tokyo, Japan). The viral solution was then concentrated by centrifugation $\left(60,000 \times g\right.$ at $4^{\circ} \mathrm{C}$ for $\left.2 \mathrm{~h}\right)$. After careful removal of the supernatant, the viral pellet was suspended in $50 \mathrm{mM}$ Tris- $\mathrm{HCl}$ buffer ( $\mathrm{pH}$ 7.8) containing $130 \mathrm{mM} \mathrm{NaCl}$ and $1 \mathrm{mM}$ EDTA. For retroviral infection, cells were plated in the wells of 24-well tissue culture plates (Greiner Bio-One, Frickenhausen, Germany) at a density of $1.5 \times 10^{4}$ cells/well. After 1 day of culture, the medium was replaced with fresh medium containing concentrated viral solutions and $8 \mu \mathrm{g} / \mathrm{mL}$ polybrene (Sigma-Aldrich). The cells were incubated at $37^{\circ} \mathrm{C}$ for $6 \mathrm{~h}$, and the medium was then changed to fresh medium. The transduction titer was determined using Hepa1-6 cells by a serial dilution method after $48 \mathrm{~h}$ of infection [11].

\subsection{Albumin secretion assay}

Hepatoma cells were plated in the wells of 24-well tissue culture plates at a density of $4.0 \times 10^{4}$ cells/well. From the next day (day 0), the cells were cultured in the presence or absence of $0.1 \mu \mathrm{g} / \mathrm{mL}$ Dox (Sigma-Aldrich). The medium was changed every other day. On day 7, the culture medium was collected and stored at $-80^{\circ} \mathrm{C}$ for subsequent analysis. The secreted albumin for $24 \mathrm{~h}$ was measured by ELISA using a commercially available kit (Mouse Albumin ELISA Quantitation Set; Bethyl, Montgomery, TX, USA) according to the manufacturer's protocol.

\subsection{Ammonia and urea metabolism analysis}

Hepatoma cells were seeded into the wells of 24 -well tissue culture plates at a density of $4.0 \times 10^{4}$ cells/well in medium with or without Dox $(0.1 \mu \mathrm{g} / \mathrm{mL})$. On day 5 , the medium was changed to fresh 
medium supplemented with $2 \mathrm{mM} \mathrm{NH}_{4} \mathrm{Cl}$ (Wako Pure Chemical Industries) and the cells were incubated for a further $6 \mathrm{~h}$. The culture medium was then collected and used for ammonia and urea analyses. The ammonia and urea concentrations in the medium were determined using commercially available kits (Ammonia-Test Wako and Urea Nitrogen-Test, respectively; Wako Pure Chemical Industries) according to the manufacturer's protocols.

\subsection{Cytochrome P450 assay}

Hepatoma cells were seeded into the wells of 24 -well tissue culture plates at a density of $4.0 \times 10^{4}$ cells/well in medium with or without Dox $(0.1 \mu \mathrm{g} / \mathrm{mL})$. On day 5 , the cytochrome P450 (CYP3A) activity of the cells was measured using an assay kit (P450-Glo ${ }^{\mathrm{TM}}$ CYP3A4 Assay with Luciferin-IPA; Promega, Madison, WI, USA) according to the manufacturer's protocols.

\subsection{Gene expression analysis by RT-PCR}

Total RNA was extracted from the cells on day 5 using a commercially available kit (RNAiso plus). RNA was reverse-transcribed into cDNA with an oligo-dT primer and ReverTra Ace reverse transcriptase. To analyze the expression of liver-specific genes, PCR amplifications were performed using G-Taq DNA polymerase (Cosmo Genetech, Seoul, Korea) at $95^{\circ} \mathrm{C}$ for $2 \mathrm{~min}$, followed by 20-40 cycles of amplification at $95^{\circ} \mathrm{C}$ for $20 \mathrm{~s}, 55^{\circ} \mathrm{C}$ for $40 \mathrm{~s}$ and $72^{\circ} \mathrm{C}$ for $10 \mathrm{~s}$, and a final extension at $72^{\circ} \mathrm{C}$ for $5 \mathrm{~min}$. Specific primers for $\alpha$-fetoprotein (AFP), transthyretin (TTR), albumin (ALB), glucose-6-phosphatase (G6P), tyrosine aminotransferase (TAT), cytochrome P450-3a (CYP3a) and glyceraldehyde-3-phosphate dehydrogenase (GAPDH) were used. The primer sequences are listed in Supplementary Table 2. To analyze the expression of exogenous LETF genes, PCR amplifications were performed using G-Taq DNA polymerase at $95^{\circ} \mathrm{C}$ for $2 \mathrm{~min}$, followed by 30 cycles of amplification at $95^{\circ} \mathrm{C}$ for $20 \mathrm{~s}, 55^{\circ} \mathrm{C}$ for $40 \mathrm{~s}$ and $72^{\circ} \mathrm{C}$ for $10 \mathrm{~s}$, and a final extension at $72^{\circ} \mathrm{C}$ for 5 min. These amplifications used the forward primers listed in Supplementary Table 3 and the reverse 
primer 5'-AGGTTGATTATCGATGCGGC-3', which anneals to the WPRE sequence. The mRNA expression levels were normalized by the mRNA expression of the housekeeping gene GAPDH. At the same time, no amplification derived from contaminated genomic DNA was confirmed by PCR using non-reverse-transcribed samples and primers annealing to GAPDH.

\subsection{Cell morphology analysis}

Hepatoma cells were seeded into the wells of 24 -well tissue culture plates at a density of $4.0 \times 10^{4}$ cells/well in medium with or without Dox $(0.1 \mu \mathrm{g} / \mathrm{mL})$. On day 5 , the cells were washed with phosphate-buffered saline (PBS) and fixed in 4\% paraformaldehyde for $15 \mathrm{~min}$. The cells were washed three times with PBS and incubated with PBS containing 4',6-diamidino-2-phenylindole (DAPI; Roche Applied Science, Mannheim, Germany) for 30 min. After three washes, the cells were observed under a BZ-9000 fluorescence microscope (Keyence, Tokyo, Japan).

\subsection{Cell proliferation analysis}

Hepatoma cells were seeded into the wells of 6-well tissue culture plates (Greiner Bio-One) at a density of $2.0 \times 10^{5}$ cells/well in medium with or without Dox $(0.1 \mu \mathrm{g} / \mathrm{mL})$. The cells were passaged every other day and the numbers of cells were counted by the Trypan blue dye staining method using a hemocytometer on days $0,3,5$ and 7 in triplicate.

\section{Results}

\subsection{Enhanced albumin secretion in hepatoma cells by inducible expression of LETF genes}

In our preliminary experiment involving infections of retroviral vectors encoding constitutive expression cassettes for LETF genes into Hepa1-6 cells, the cell growth was suppressed with some combinations of vectors. Therefore, in this study, a tetracycline-inducible gene expression system was incorporated into a retroviral vector system for inducible expression of the LETF genes. The 
system consisted of a combination of two retroviral vectors, one for constitutively expressing rtTA that can be activated by Dox addition and the other for expressing an LETF gene under the control of an inducible promoter containing a binding site (TRE) for activated rtTA. To make the system work effectively in the cells, we first established a founder cell line that constantly expressed rtTA. After the retroviral vector encoding the expression cassette for rtTA (Fig. 1A) was infected into Hepa1-6 cells, EGFP-positive Hepa1-6 cell clones were established by the limiting dilution method. To confirm the inducible expression of a target gene by rtTA activation, a retroviral vector produced from pQMSCV/EGFP-TREtight-DsRed-WPRE, in which the DsRed gene is expressed under the control of the TREtight promoter (Fig. 1A), was infected into the cells, and the cells were cultured with or without Dox for 2 days. A cell line in which the DsRed expression could be strictly controlled by Dox addition was established (Hepa/rtTA) (Fig. 1B), and used for subsequent experiments.

Next, Hepa/rtTA cells were infected with retroviral vectors encoding the individual LETF genes (Fig. 1A) at a multiplicity of infection (MOI) of 13.5 for each vector, and the infected cells were cultured in medium with or without Dox (Dox+ and Dox-, respectively). To evaluate the expression of liver functions induced by LETF overexpression, the albumin secretion rate of the cells was measured on day 7 (Fig. 1C). When the LETF genes were separately transduced into the cells, enhanced albumin secretion rates after Dox addition were observed for HNF-3 $\beta$, HNF-6 and C/EBP- $\beta$. However, when all three LETF genes (HNF-3 $\beta$, HNF-6 and C/EBP- $\beta$ ) were transduced into Hepa/rtTA cells, no further enhancement of the albumin secretion rate was observed. When all eight LETF genes were transduced into Hepa/rtTA cells, the cells exhibited a significantly enhanced albumin secretion rate after Dox addition.

\subsection{Analyses of liver functions for Hepa/8F clones}


A retroviral vector heterogeneously infects cells and randomly inserts a transgene into the cell genome. Therefore, retroviral-transduced cells without cloning are considered to be a mixture of cells with various expression levels of the transgene, and to show the average expression level. Therefore, we speculated that we might be able to isolate the cells with the highest expression of liver functions after induction from the 8-LETF transduced Hepa/rtTA (Hepa/8F) cells. Many clones were established from Hepa/8F cells by the limiting dilution method. Among them, 20 clones were randomly picked up, and their albumin secretion rates were measured with or without LETF gene induction by Dox. As shown in Fig. 2A, most of the Hepa/8F clones upregulated their albumin secretion rate after Dox addition, but the enhancement levels differed among the clones. In particular, remarkably enhanced rates of albumin secretion were observed for clones \#4, \#5, \#16 and \#17 (Fig. 2A). The ammonia removal ability as another liver function was measured for the Hepa/8F clones on day 5 of culture with or without Dox (Fig. 2B). The ammonia removal rates for Hepa1-6, Hepa/rtTA and Hepa/8F cells showed negative values with and without Dox, indicating ammonia production that possibly arose through amino acid metabolism. The production level was diminished for the Hepa/8F clones with Dox addition. In Hepa/8F clone \#5 cell cultures with Dox, ammonia was significantly reduced in the medium and the urea synthesis rate was increased $(0.014 \pm 0.005$ and $0.050 \pm 0.018 \mathrm{pmol} /(\mathrm{cell} \cdot \mathrm{h})$ for Dox- and Dox + , respectively). Furthermore, the cytochrome P450 (CYP3A) activity was measured for the Hepa/8F clones on day 5 of culture with or without Dox (Fig. 2C). The highest level of CYP3A activity comparable to that of primary hepatocytes $(2.14 \pm 0.13$ RLU/cell) was observed for Hepa/8F clone \#5 with Dox addition.

Liver-specific gene expressions (AFP, TTR, G6P, TAT, ALB and CYP3a) in Hepa/8F clones \#4, $\# 5$, \#16 and \#17, which showed high rates of albumin secretion after induction by Dox, were analyzed by RT-PCR on day 5 of culture with or without Dox (Fig. 2D). Owing to the nature of hepatoma cells, AFP was expressed in all the cell lines. The AFP expression level was enhanced by Dox addition to the Hepa/8F clones. The liver-specific gene expressions other than AFP were not 
detected in parental hepatoma cells (Hepa1-6 and Hepa/rtTA cells). These gene expressions were induced by Dox addition to the Hepa/8F clones, although the induction levels differed among the clones. The highest levels of gene induction were observed for clone \#5.

\subsection{Morphological observation and proliferation of Hepa/8F cells}

The cell morphologies were observed under culture conditions with or without Dox (Fig. 3A). The parental Hepa1-6 and Hepa/rtTA cells remained unchanged with or without Dox. Upon overexpression of LETFs by Dox addition to Hepa/8F cells, the cell morphology changed to a more epithelial-like morphology, with strong growth arrest. The morphological change was significant for clone \#5. The cell size of clone \#5 became large after the induction, and binucleated cells were also observed during the culture, as seen in cultured primary hepatocytes.

Hepa/8F cells showed strong growth arrest after induction by Dox. Therefore, growth curves for Hepa1-6, Hepa/rtTA and Hepa/8F(\#5) cells were measured under the culture conditions with or without Dox addition (Fig. 3B). The growth of parental Hepa1-6 and Hepa/rtTA cells was not affected by Dox addition. In contrast, the growth of $\mathrm{Hepa} / 8 \mathrm{~F}(\# 5)$ cells was inhibited after induction by Dox from the early culture period, and the cell number remained nearly constant during the culture period. Nevertheless, no obvious apoptotic cells were observed in the Hepa/8F(\#5) culture with Dox addition.

\subsection{Expression levels of exogenous LETF genes in Hepa/8F cells}

The expression levels of the exogenous LETF genes in Hepa/8F clones \#4, \#5, \#16 and \#17 were analyzed by RT-PCR on day 5 of culture with or without Dox (Fig. 4). The expression levels differed among the clones. Clone \#5 cells expressed the exogenous LETF genes mostly at higher levels than the other clones, whereas the other clones expressed one or more LETF genes at very low levels. Genomic PCR analyses confirmed that all the LETF genes were introduced into the cell genome of 
clone \#5 cells by the retroviral vectors, while one of the eight LETF genes was not integrated into the genome in the other clones (data not shown).

\section{Discussion}

For the construction of BAL systems, the use of effective cells with high liver functions is the most important factor determining system performance. Although the cells used in BAL systems are desired to grow in ordinary cell culture, the abilities of both proliferation and high liver functions are often incompatible. Thus, the development of cells capable of switching between proliferation and differentiation is required. In this study, we successfully established a genetically engineered hepatoma cell line, Hepa/8F(\#5), in which enhanced liver functions could be induced by overexpression of eight LETF genes via Dox addition. Hepa/8F(\#5) cells grew easily in normal culture medium. After induction using Dox-containing medium, Hepa/8F(\#5) cells immediately ceased growth and exhibited significant albumin secretion and metabolic capabilities of ammonia removal and urea synthesis. Furthermore, the cytochrome P450 activity was considerably induced, representing mature hepatocyte features. The albumin secretion was enhanced by 50 -fold and this secretion level was comparable to that in cultured primary hepatocytes $[12,13]$. The ammonia removal ability was almost lost in parental Hepa1-6 cells, while this important hepatic function was induced in Hepa/8F(\#5) cells after Dox addition. The levels of liver functions for Hepa/8F(\#5) cells after Dox addition are summarized in Table 1. Although the abilities of albumin secretion and ammonia removal induced by overexpression of the LETF genes in Hepa/8F(\#5) cells were slightly lower than those of freshly isolated mouse primary hepatocytes, the level of cytochrome P450 (CYP3A) activity was comparable to that of hepatocytes.

Liver-specific genes have been categorized into several groups representing the stages of hepatocyte differentiation. ALB, G6P and TAT were categorized as perinatal hepatocyte markers [14] and CYP3a was categorized as a mature hepatocyte marker [15]. Although the expressions of 
these middle- and late-stage marker genes were not detected in Hepa1-6 and Hepa/rtTA cells, they were strongly induced in $\mathrm{Hepa} / 8 \mathrm{~F}(\# 5)$ cells by overexpression of the LETF genes. These findings indicate a high degree of hepatic maturation of Hepa/8F(\#5) cells after induction by Dox.

In the expression analysis of the exogenous LETF genes in the Hepa/8F clones, the expression profiles differed among the clones. Hepa/8F(\#5) cells expressed all the LETF genes at high levels, whereas one or more LETF genes were expressed at low levels in the other clones. These differences in the expression profiles of the LETF genes might be responsible for the high liver functions in Hepa/8F(\#5) cells. In the liver, a complex regulatory mechanism is involved in the expression of liver functions. LETFs regulate not only their target genes related to liver functions but also their own and/or mutual genes, forming a transcriptional network to express and maintain the hepatic phenotype [16-18]. The expression profiles and levels of LETFs are regulated appropriately in cells through this network and the cell phenotype is highly dependent on the expression profiles of these transcription factors. In the present study, HNF-3 $\beta$, HNF-6 and C/EBP- $\beta$ individually enhanced the albumin secretion by hepatoma cells, but the combination of the three LETFs did not further enhance the ability. On the other hand, after gene transduction of all eight LETFs, the liver function was further enhanced, indicating that a suitable combination and balanced expression of LETFs are important for expression of the highest level of liver functions in hepatoma cells. Thus, the detailed expression profiles of the LETFs in Hepa/8F(\#5) cells before and after Dox induction should be elucidated to determine the transcriptional network in the hepatoma cells.

In this study, the cells were cultured in monolayers using ordinary culture medium without any addition of cytokines for differentiation. Under these culture conditions, Hepa/8F(\#5) cells expressed the highest level of liver functions. It has been reported that parenchymal hepatocytes and hepatoma cells exhibited high liver functions in three-dimensional cultures such as spheroids and organoids $[19,20]$. Thus, an effective culture medium and culture method may make it possible to further improve the liver functions in $\mathrm{Hepa} / 8 \mathrm{~F}(\# 5)$ cells. 


\section{Conclusion}

In the present study, we have shown that overexpression of LETFs including HNFs and C/EBPs enabled the induction of enhanced liver functions in hepatoma cells. Switching between proliferation and expression of differentiated functions could be controlled by the inducible expression of LETFs in the genetically engineered hepatoma cells. This genetic modification method for hepatoma cells can provide a means for generating cell sources for basic hepatic cell research and applied fields such as the construction of BAL systems and drug testing studies using liver functions.

\section{Acknowledgment}

This work was supported in part by a Grant-in-Aid for Scientific Research (no. 23650289) from the Japan Society for the Promotion of Science.

\section{References}

[1] S. Bellentani, C. Tiribelli, The spectrum of liver disease in the general population: lesson from the Dionysos study, J. Hepatol. 35 (2001) 531-537.

[2] J. Neuberger, Liver transplantation, J. Hepatol. 32 (2000) 198-207.

[3] J.K. Park, D.H. Lee, Bioartificial liver systems: current status and future perspective, J. Biosci. Bioeng. 99 (2005) 311-319.

[4] N. Kobayashi, T. Okitsu, S. Nakaji, N. Tanaka, Hybrid bioartificial liver: establishing a reversibly immortalized human hepatocyte line and developing a bioartificial liver for practical use, J. Artif. Organs 6 (2003) 236-244.

[5] H. Schrem, J. Klempnauer, J. Borlak, Liver-enriched transcription factors in liver function and development. Part I: the hepatocyte nuclear factor network and liver-specific gene expression, Pharmacol. Rev. 54 (2002) 129-158. 
[6] S. Cereghini, Liver-enriched transcription factors and hepatocyte differentiation, FASEB J. 10 (1996) 267-282.

[7] M. Nagaki, H. Moriwaki, Transcription factor HNF and hepatocyte differentiation, Hepatol. Res. 38 (2008) 961-969.

[8] R.G. Hawley, F.H. Lieu, A.Z. Fong, T.S. Hawley, Versatile retroviral vectors for potential use in gene therapy, Gene Ther. 1 (1994) 136-138.

[9] H. Akiyama, A. Ito, Y. Kawabe, M. Kamihira, Genetically engineered angiogenic cell sheets using magnetic force-based gene delivery and tissue fabrication techniques, Biomaterials 31 (2010) 1251-1259.

[10] C.A. Penno, Y. Kawabe, A. Ito, M. Kamihira, Production of recombinant human erythropoietin/Fc fusion protein by genetically manipulated chickens, Transgenic Res. 19 (2010) 187-195.

[11] A. Hotta, Y. Saito, K. Kyogoku, Y. Kawabe, K. Nishijima, M. Kamihira, S. Iijima, Characterization of transient expression system for retroviral vector production, J. Biosci. Bioeng. 101 (2006) 361-368.

[12] R. Iacob, U. Rüdrich, M. Rothe, S. Kirsch, B. Maasoumy, N. Narain, C.M. Verfaillie, P. Sancho-Bru, M. Iken, I. Popescu, A. Schambach, M.P. Manns, M. Bock, Induction of a mature hepatocyte phenotype in adult liver derived progenitor cells by ectopic expression of transcription factors, Stem Cell Res. 6 (2011) 251-261.

[13] K. Yamada, M. Kamihira, S. Iijima, Enhanced cell aggregation and liver functions using polymers modified with a cell-specific ligand in primary hepatocyte cultures, J. Biosci. Bioeng. 88 (1999) 557-562.

[14] B.A. Haber, S. Chin, E. Chuang, W. Buikhuisen, A. Naji, R. Taub, High-Levels of Glucose-6-Phosphatase Gene and Protein Expression Reflect an Adaptive Response in Proliferating Liver and Diabetes, J. Clin. Invest. 95 (1995) 832-841. 
[15] C. Noda, C. Fukushima, T. Fujiwara, K. Matsuda, Y. Kobune, A. Ichihara, Developmental regulation of rat serine dehydratase gene expression: evidence for the presence of a repressor in fetal hepatocytes, Biochim. Biophys. Acta. 1217 (1994) 163-173.

[16] J. Li, G. Ning, S.A. Duncan, Mammalian hepatocyte differentiation requires the transcription factor HNF-4alpha. Genes Dev. 14 (2000) 464-474.

[17] F. Parviz, C. Matullo, W.D. Garrison, Hepatocyte nuclear factor 4 alpha controls the development of a hepatic epithelium and liver morphogenesis, Nat. Genet. 34 (2003) 292-296.

[18] C. Yin, Y. Lin, X. Zhang, Y.X. Chen, X. Zeng, H.Y. Yue, J.L. Hou, X. Deng, J.P. Zhang, Z.G. Han, W.F. Xie, Differentiation therapy of hepatocellular carcinoma in mice with recombinant adenovirus carrying hepatocyte nuclear factor-4alpha gene, Hepatology 48 (2008) 1528-1539.

[19] R. Hamamoto, K. Yamada, M. Kamihira, S. Iijima, Differentiation and proliferation of primary rat hepatocytes cultured as spheroids, J. Biochem. 124 (1998) 972-979.

[20] J. Fukuda, K. Okamura, K. Ishihara, H. Mizumoto, K. Nakazawa, H. Ijima, T. Kajiwara, K. Funatsu, Differentiation effects by the combination of spheroid formation and sodium butyrate treatment in human hepatoblastoma cell line (Hep G2): a possible cell source for hybrid artificial liver, Cell Transplant. 14 (2005) 819-827. 


\section{Figure legends}

Fig. 1. Inducible expression of the LETF genes in Hepa1-6 cells using retroviral vectors for gene transfer. (A) Constructs of the retroviral vectors encoding the rtTA, DsRed and LETF genes. (B) Induction of gene expression by Dox in the rtTA-expressing founder cell line (Hepa/rtTA). Hepa/rtTA cells infected by a retroviral vector encoding DsRed under the control of the TREtight promoter were cultured in the absence or presence of Dox (Dox- and Dox + , respectively). After 2 days of culture, the cells were photographed under bright-field (upper) and fluorescence (lower) microscopy. Scale bars: $200 \mu \mathrm{m}$. (C) Albumin secretion rates of LETF gene-transduced cells on day 7 of culture in the absence (open columns) or presence (closed columns) of Dox. 3-LETFs: cells transduced with HNF-3 $\beta$, HNF-6 and C/EBP- $\beta$; 8-LETFs: cells transduced with all eight LETFs at the same time; DsRed, cells transduced with DsRed as a control; mock, cells not infected by retroviral vectors. The data shown are means $\pm \mathrm{SD}(n=3) . * P<0.05, * * P<0.01$ vs. Dox- by Student's $t$-test.

Fig. 2. Analyses of liver functions and liver-specific gene expressions of Hepa/8F clones. (A-C) Liver function analyses. The albumin secretion rates of the $20 \mathrm{Hepa} / 8 \mathrm{~F}$ clones were measured on day 7 of culture (A). Hepa1-6, Hepa/rtTA, Hepa/8F (8F-transduced bulk cells) and established Hepa/8F clones (\#4, \#5,\#16 and \#17) were analyzed for their ammonia removal rates (B) and CYP3A activities (C) on day 5 of culture. The cells were cultured in the absence (open columns) or presence (closed columns) of Dox. The data shown are means $\pm \operatorname{SD}(n=3)$. ${ }^{*} P<0.05, * * P<0.01$ vs. Dox- by Student's t-test. (D) RT-PCR analysis for the expressions of liver-specific genes in Hepa1-6, Hepa/rtTA and Hepa/8F clones (\#4, \#5, \#16 and \#17) on day 5 of culture. The mRNA expression levels were normalized by the mRNA expression level of the housekeeping gene GAPDH. 
Fig. 3. Morphological observation and proliferation of Hepa/8F(\#5) cells. (A) Bright-field [Hepa1-6, Hepa/rtTA and Hepa/8F(\#5)] and fluorescence $[\mathrm{Hepa} / 8 \mathrm{~F}(\# 5)]$ photographs of cells after 5 days of culture in the absence or presence of Dox (Dox- and Dox + , respectively). EGFP expression images (green) representing the shapes of cells and DAPI-staining images (blue) were merged in fluorescence photographs. Scale bars: $50 \mu \mathrm{m}$. (B) Cell proliferation of Hepa1-6, Hepa/rtTA and Hepa/8F(\#5) cells during 7 days of culture in the absence (open circles) or presence (closed circles) of Dox. The data are means $\pm \mathrm{SD}(\mathrm{n}=3) .{ }^{*} \mathrm{P}<0.01$ vs. Dox- by Student's $t$-test.

Fig. 4. RT-PCR analyses for the expression of exogenous LETF genes. Hepa1-6, Hepa/rtTA and Hepa/8F clones (\#4, \#5, \#16 and \#17) were measured for the expression of the eight exogenous LETF genes after 5 days of culture in the absence or presence of Dox (Dox- and Dox + , respectively). The mRNA expression levels were normalized by the mRNA expression level of the housekeeping gene GAPDH. 
Table 1. Comparative representation of liver functions for Hepa1-6, Hepa/8F(\#5) $[\mathrm{Dox}+]$ and mouse primary hepatocytes.

\begin{tabular}{cccc}
\hline Cells & $\begin{array}{c}\text { Albumin secretion rate } \\
{[\mathrm{pg} /(\text { cell·day })]}\end{array}$ & $\begin{array}{c}\text { Ammonia removal rate } \\
{[\mathrm{pmol} /(\mathrm{cell} \cdot \mathrm{h})]}\end{array}$ & $\begin{array}{c}\text { P450 }(\mathrm{CYP} 3 \mathrm{~A}) \text { activity } \\
{[\mathrm{RLU} / \text { cell }]}\end{array}$ \\
\hline Hepa1-6 & $1.8 \pm 0.2$ & $-0.068 \pm 0.024$ & $0.0020 \pm 0.0001$ \\
Hepa/8F(\#5) $[$ Dox +$]$ & $90.3 \pm 7.4$ & $0.139 \pm 0.015$ & $2.00 \pm 0.26$ \\
Primary hepatocytes & $246 \pm 53$ & $0.948 \pm 0.014$ & $2.14 \pm 0.13$ \\
\hline
\end{tabular}


A

MSCV/EGFP/CMV/rtTA/WPRE

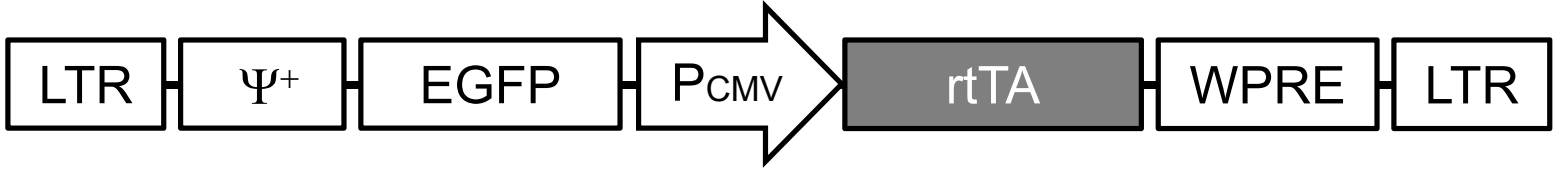

MSCV/EGFP/TRE $E_{\text {tight/DsRed or LETF gene/WPRE }}$

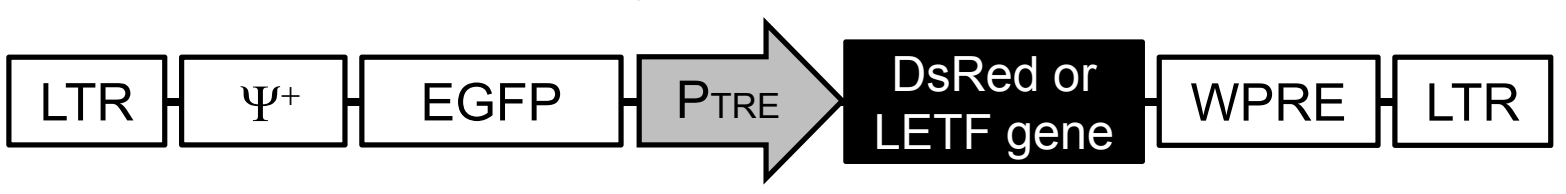

B

Dox- Dox+

Bright-field

Fluorescence

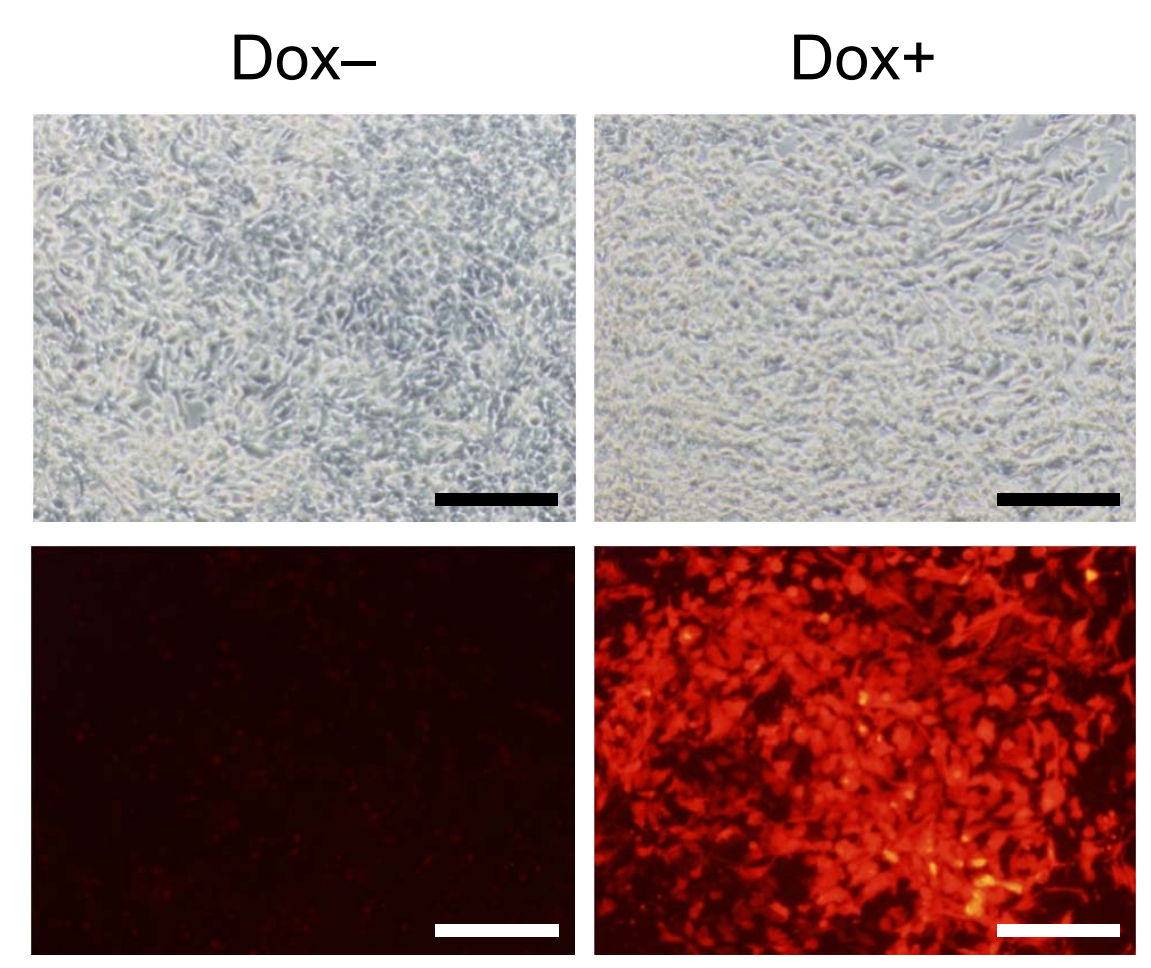

Fig. 1A, B Yamamoto et al. 
C

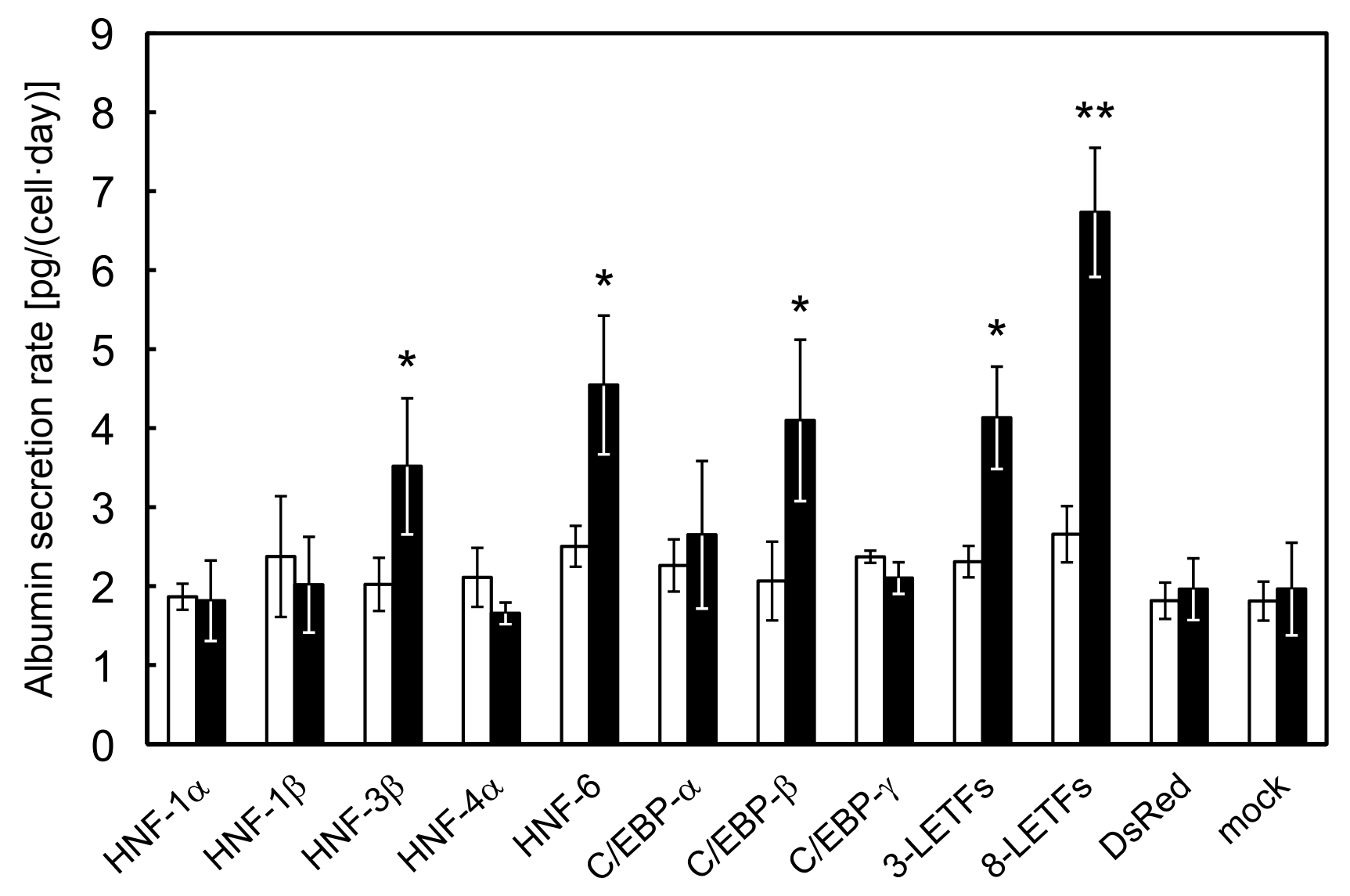

Fig. 1C Yamamoto et al. 
A



Fig. 2A Yamamoto et al. 
B

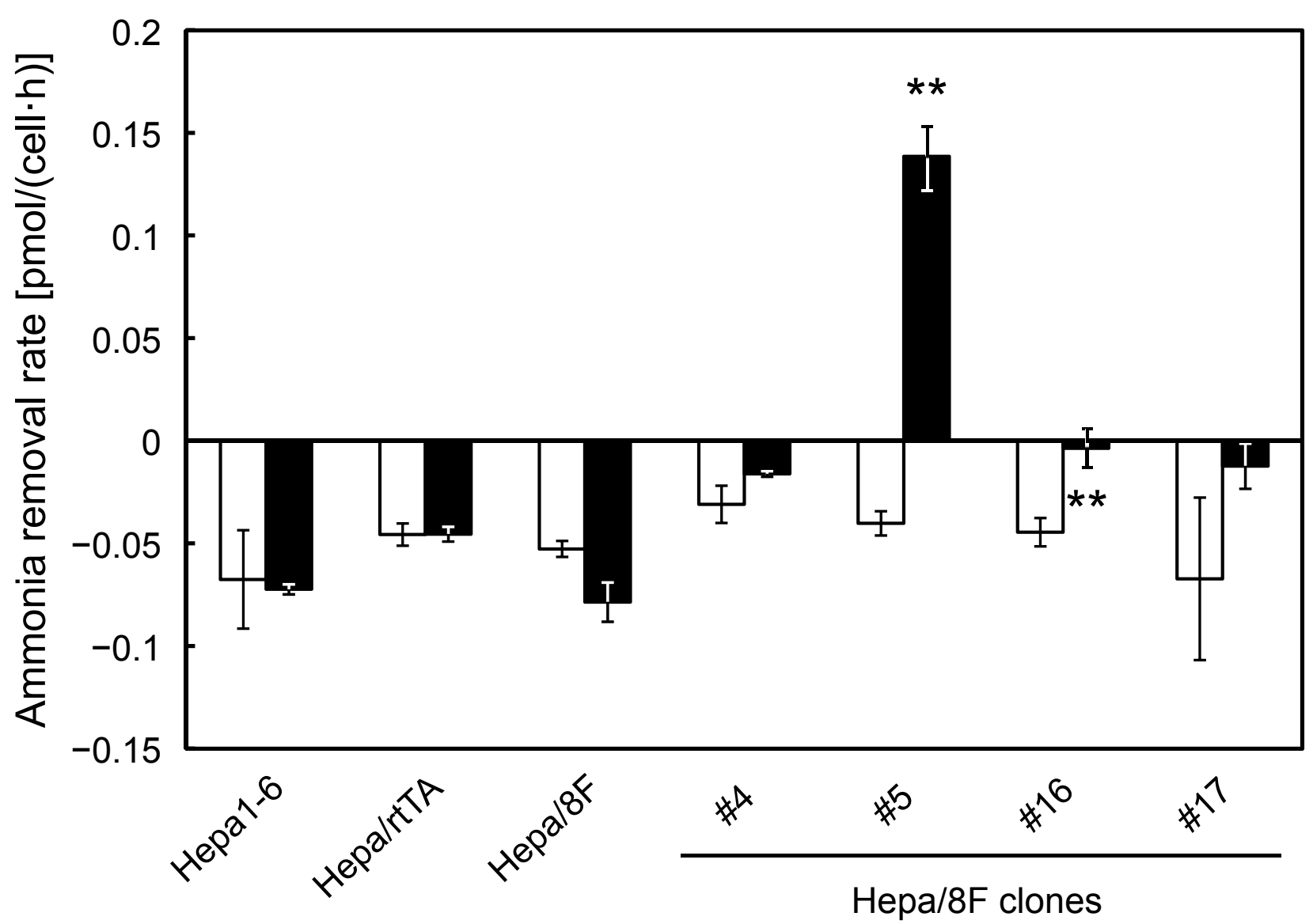

Fig. 2B Yamamoto et al. 
C



Fig. 2C Yamamoto et al. 
D

Hepa/8F clones

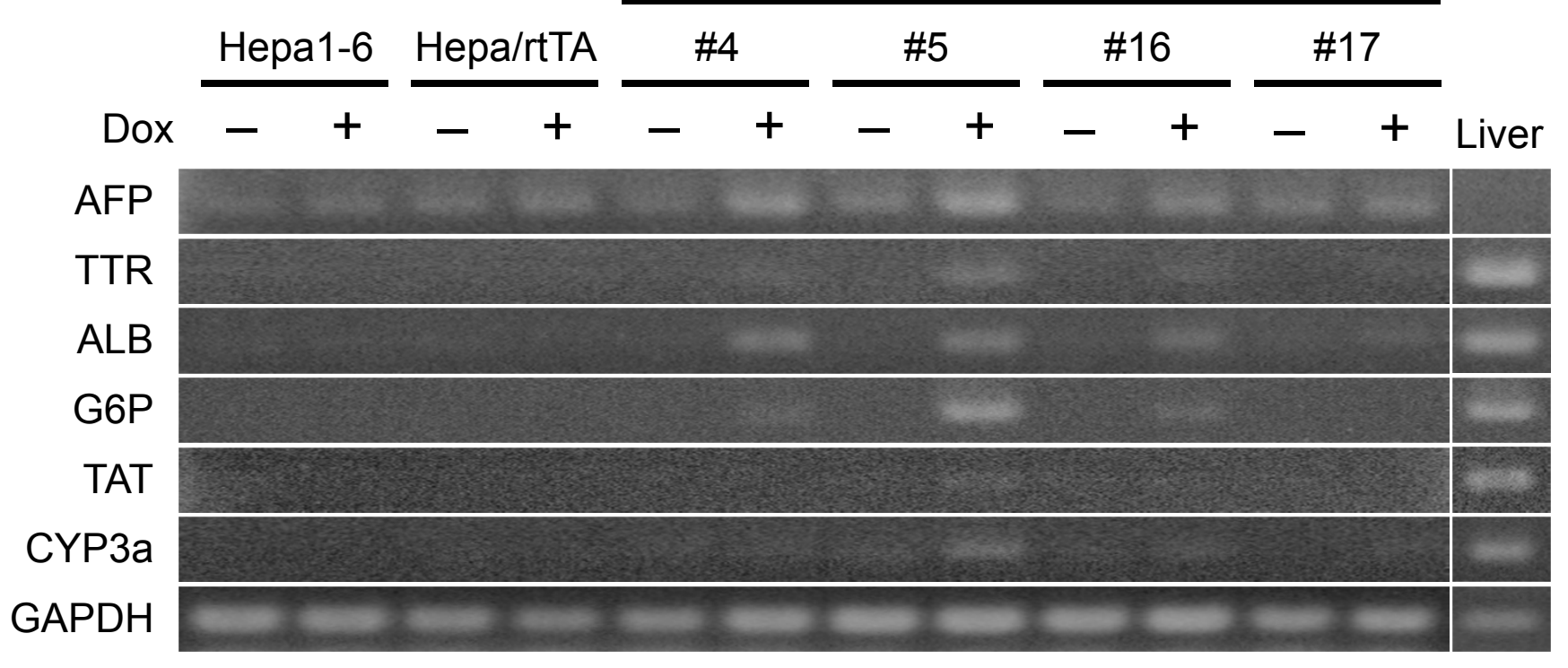

Fig. 2D Yamamoto et al. 
A

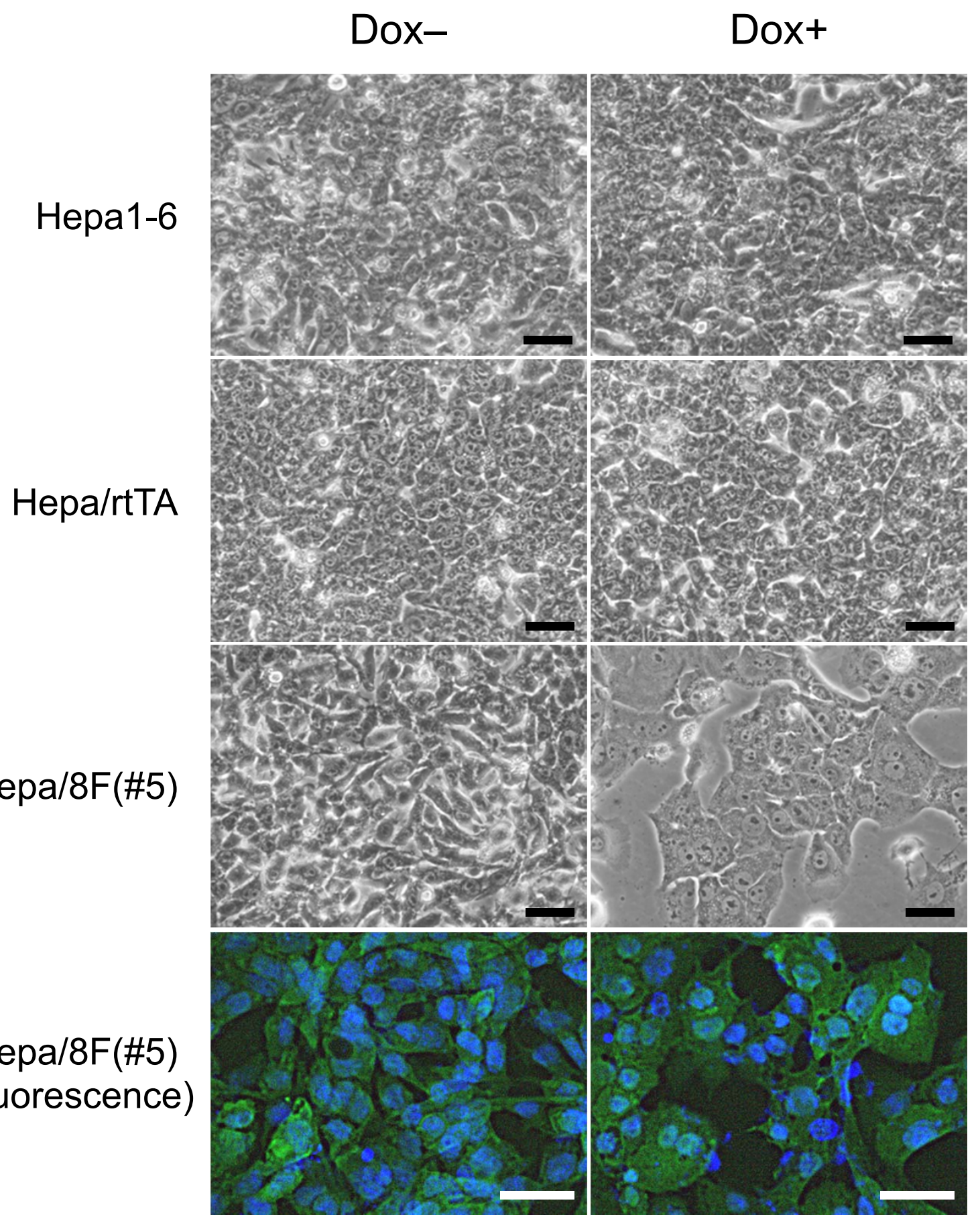

Fig. 3A Yamamoto et al. 
B
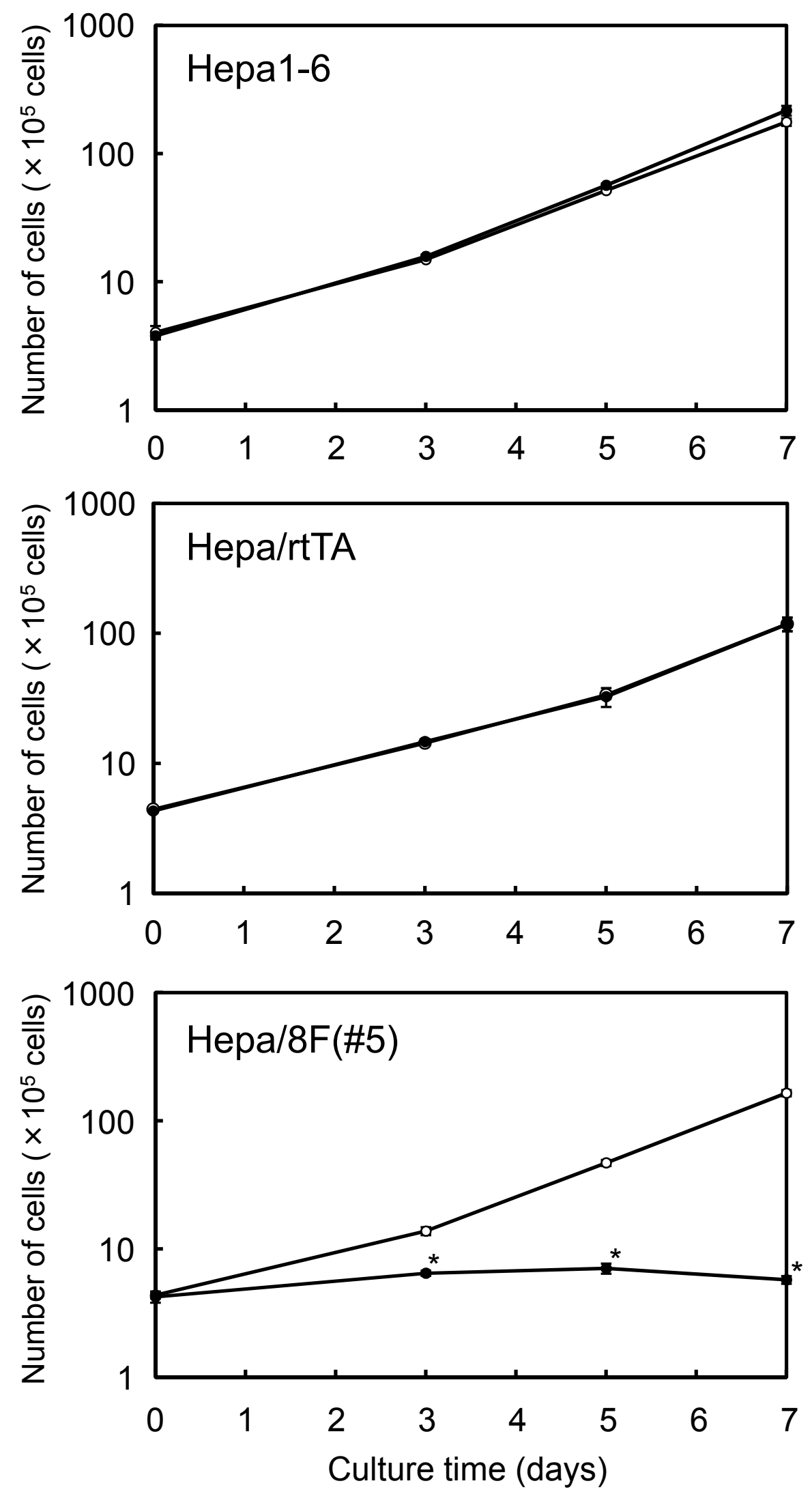

Fig. 3B Yamamoto et al. 


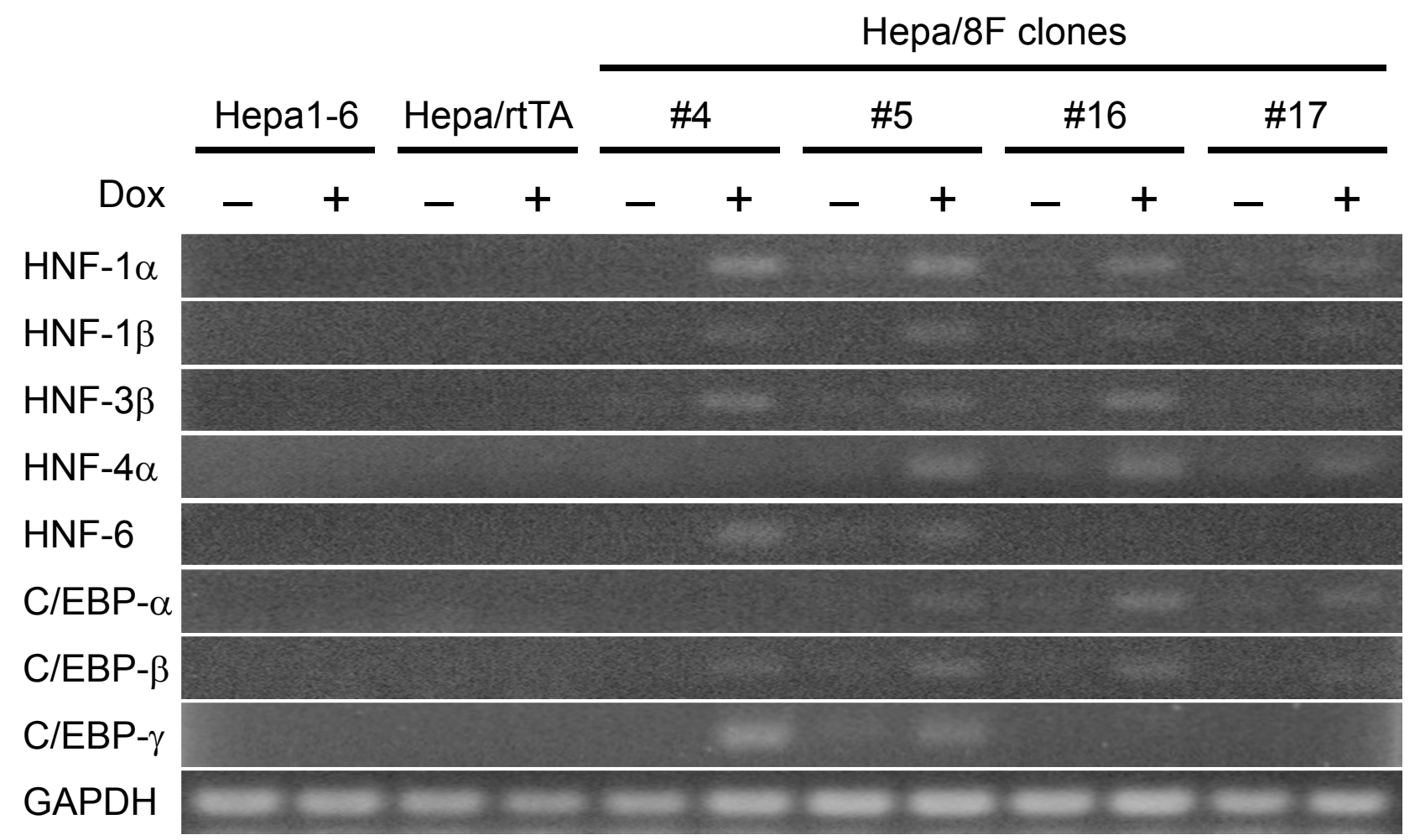

Fig. 4 Yamamoto et al. 
Supplementary Table 1. PCR primers used in cloning of LETFs (Restriction sites were underlined and annealing temperatures were described in $\operatorname{Tm}\left({ }^{\circ} \mathrm{C}\right)$ ).

\begin{tabular}{|c|c|c|c|c|}
\hline Gene & Direction & Sequence & Site & $\operatorname{Tm}$ \\
\hline \multirow{2}{*}{$\begin{array}{l}\text { HNF-1 } \alpha \\
\text { (first part) }\end{array}$} & FW & 5'-ACGCGTCGACCATGGTTTCTAAGCTGAGCCA-3' & SalI & \multirow{2}{*}{57} \\
\hline & RV & 5'-CGGTGGAGGTCCGTTATAGGTG-3' & - & \\
\hline \multirow{2}{*}{$\begin{array}{c}\text { HNF-1 } \alpha \\
\text { (latter part) }\end{array}$} & FW & 5'-GCGTGTCTACAACTGGTTTGCC-3' & - & \multirow{2}{*}{57} \\
\hline & RV & 5'-AAGGATCCACGGTTACTGGGAAGAGG-3' & BamHI & \\
\hline \multirow[t]{2}{*}{ HNF-1 $\beta$} & FW & 5'-AAACTCGAGACCATGGTGTCCAAGCTCACGTCG-3' & XhoI & \multirow{2}{*}{58} \\
\hline & RV & 5'-AAGGATCCGAGTATCACCAGGCTTGCAGTGG-3' & Bam HI & \\
\hline \multirow[t]{2}{*}{ HNF-3 $\beta$} & FW & 5'-AAACTCGAGACCATGCTGGGAGCCGTGAAGAT-3' & XhoI & \multirow{2}{*}{58} \\
\hline & RV & 5'-AAGGATCCGCTAGCAGGGCCTGAAAGCC-3' & BamHI & \\
\hline \multirow[t]{2}{*}{$\mathrm{HNF}-4 \alpha$} & FW & 5'-ACGCGTCGACCATGCGACTCTCTAAAACCCTT-3' & SalI & \multirow{2}{*}{48} \\
\hline & $\mathrm{RV}$ & 5'-CGGGATCCTTGCTAGATGGCTTCTTGCT-3' & BamHI & \\
\hline \multirow[t]{2}{*}{ HNF-6 } & FW & 5'-AAACTCGAGACCATGAACGCACAGCTGACCAT-3' & XhoI & \multirow{2}{*}{48} \\
\hline & RV & 5'-CGGGATCCCTCATGCTTTGGTACAAG-3' & BamHI & \\
\hline \multirow[t]{2}{*}{ C/EBP- $\alpha$} & FW & 5'-TTTCTCGAGACCATGGAGTCGGCCGACTTCTA-3' & XhoI & \multirow{2}{*}{61} \\
\hline & $\mathrm{RV}$ & 5'-AAGGATCCTCACGCGCAGTTGCCC-3' & BamHI & \\
\hline \multirow[t]{2}{*}{$\mathrm{C} / \mathrm{EBP}-\beta$} & FW & 5'-AAACTCGAGACCATGCACCGCCTGCTGGCCTG-3' & XhoI & \multirow{2}{*}{65} \\
\hline & RV & 5'-AAGGATCCGCCGCGCTAGCAGTGGCCCG-3' & BamHI & \\
\hline \multirow[t]{2}{*}{$\mathrm{C} / \mathrm{EBP}-\gamma$} & FW & 5'-AAACTCGAGACCATGAGCAAGCTGTCGCAGCCA-3' & XhoI & \multirow{2}{*}{61} \\
\hline & RV & 5'-CGGGATCCTACTGCCCTGGGTTATCAGAATTTGTC-3' & BamHI & \\
\hline
\end{tabular}


Supplementary Table 2. Primers used in RT-PCR analysis for liver-specific genes.

\begin{tabular}{cccc}
\hline Gene & Direction & Sequence & Product size (bp) \\
\hline AFP & FW & 5'-TCGTATTCCAACAGGAGG-3' & 174 \\
& RV & 5'-AGGCTTTTGCTTCACCAG-3' & \\
TTR & FW & 5'-AAAGTCCTGGATGCTGTCCG-3' & 142 \\
& RV & 5'-CATCTGTGGTGAGCCCGTG-3' & 122 \\
ALB & FW & 5'-CCCAACTCTCGTGGAGGC-3' & \\
& RV & 5'-CACACACGGTTCAGGATTGC-3' & 160 \\
G6P & FW & 5'-GTGTCTGTGATTGCTGACCTGAG-3' & \\
& RV & 5'-AATACGGGCGTTGTCCAAAC-3' & 208 \\
TAT & FW & 5'-AAACCTTCAATCCCATCCGA-3' & \\
& RV & 5'-TCCCGACTGGATAGGTAGCC-3' & 124 \\
CYP3a & FW & 5'-TACTTGAGGCAGAAGGCAAAGA-3' & \\
& RV & 5'-CTGGGTTGTTGAGGGAATCC-3' & \\
GAPDH & FW & 5'-CTACCCCCAATGTGTCCGTC-3' & \\
& RV & 5'-GCTGTTGAAGTCGCAGGAGAC-3' & \\
\hline
\end{tabular}


Supplementary Table 3. Primers used in RT-PCR analysis for exogenous LETF genes.

\begin{tabular}{cccc}
\hline Gene & Direction & Sequence & Product size (bp) \\
\hline HNF-1 $\alpha$ & FW & 5'-GCACCAGTCCCACAGTGTCC-3' & 197 \\
HNF-1 $\beta$ & FW & 5'-CCCCCCAGTATTCCCACAC-3' & 177 \\
HNF-3 $\beta$ & FW & 5'-CAGTCACGAACAAAGCGGG-3' & 177 \\
HNF-4 $\alpha$ & FW & 5'-CACCAAGTGGCTCGGGAT-3' & 172 \\
HNF-6 & FW & 5'-GACAAGTGGCAGGACGAGG-3' & 129 \\
C/EBP- $\alpha$ & FW & 5'-GGAGTTGACCAGTGACAATGACC-3' & 188 \\
C/EBP- $\beta$ & FW & 5'-AGCACCCTGCGGAACTTG-3' & 125 \\
C/EBP- $\gamma$ & FW & 5'-AAGGAAGAGAATGAACGGTTGG-3' & 214 \\
\hline
\end{tabular}

